\title{
Influence of Loading Speed on Acoustic Emission During Destruction of a Composite by Von Mises Criterion
}

\author{
Sergii Filonenko ${ }^{1}$, Viacheslav Stadychenko ${ }^{2, *}$ \\ ${ }^{1}$ Department of Computerized Electrical Systems and Technology, Aerospace Faculty, National Aviation University, Kyiv, Ukraine \\ ${ }^{2}$ Department of Hydro-Gas Systems, Aerospace Faculty, National Aviation University, Kyiv, Ukraine
}

\section{Email address:}

Stadnychenko1971@gmail.com (V. Stadychenko)

${ }^{*}$ Corresponding author

\section{To cite this article:}

Sergii Filonenko, Viacheslav Stadychenko. Influence of Loading Speed on Acoustic Emission During Destruction of a Composite by Von Mises Criterion. American Journal of Mechanical and Materials Engineering. Vol. 4, No. 3, 2020, pp. 54-49.

doi: $10.11648 /$ j.ajmme.20200403.13

Received: March 12, 2020; Accepted: April 14, 2020; Published: August 31, 2020

\begin{abstract}
In this study of the composite material's destruction caused by transverse force with the use of von Mises criterion, the simulation outcomes of the composite material's cells destruction process and generated AE signals with increasing the strain rate is considered. The time dependencies of the change in the number of remaining elements and the generated AE signals with increasing the strain rate are obtained. Simulation outcomes of the process of composite cells destruction by von Mises criterion and generated acoustic emission signals with increasing strain rate are presented. It was determined that with increasing strain rate of the composite, there is an increase of decline rate of the strain curve of the remaining cells in time and a decrease in time of the destruction process. It was found that with increasing strain rate of the composite, the amplitude of the generated acoustic emission signal increases while its duration decreases, along with gradual transformation of the acoustic emission signal into a triangular signal. It was determined that with increasing strain rate of the composite, the pattern of change in the maximum amplitude of the generated acoustic emission signals has a linear character of increase, and their duration - nonlinear character of decline.
\end{abstract}

Keywords: Composite, Destruction, Acoustic Emission, Threshold Stress, Signal Amplitude, Fracture Criterion, Deformation

\section{Introduction}

Composite materials (CM) are given much attention in the scientific literature. This fully applies to the development of methods for $\mathrm{CM}$ condition verification and monitoring. High physical and mechanical characteristics of the $\mathrm{CM}$ induce their use in various products operating under high loads, temperatures and aggressive environments. One of these part elements is friction pairs. However, the tendency of the CM to brittle fracture requires continuous monitoring of products at the production stage and, especially, at the operation stage.

A wide range of studies with the use of various methods is carried out to develop methods for monitoring and diagnosing the condition of the CM products.

First of all, this applies to methods that are highly sensitive to processes of plastic deformation and fracture of materials at various levels. Such highly sensitive methods include the method of acoustic emission (AE) which is a projection of the dynamics of the processes occurring in materials when subjected to loading. However, the high sensitivity of the AE method to sub-micro, micro, and macro processes leads to the problem of interpreting the recorded information.

From this perspective, the theoretical studies of AE signal formation and emission are of great importance. Research is aimed at solving some tasks in the problem of interpreting acoustic emission.

Search for the relationship of AE signals and their parameters to the processes occurring in the material structure for the accepted models and fracture criteria. Determination of patterns of change in acoustic emission and its parameters including effects of various factors. These patterns are the basis for developing methods for monitoring and diagnosing the condition of CM products. 


\section{Analysis of Recent Research}

A great deal of attention is paid in scientific literature to study the phenomena of composite materials destruction. Studies are carried out using various methods, including the AE technique. However, most publications refer to experimental studies [1-7]. This is due to the widespread use of the $\mathrm{CM}$ in manufacturing of various structures and their components. First of all, this is applicable to critical elements subjected to significant loadings. The analysis of the research results is aimed at searching for patterns, parameters, and criteria that represent (characterize) the course of processes in the CM structure under loading - crack formation and growth, the destruction of individual elements, delamination, etc. At the same time, the predictive dependencies are constructed that are aimed to determine the behavior of the CM under load and characterize its "lifetime". As a rule, these dependencies are associated with the analysis of the accumulated energy of acoustic emission, which is due to the complexity of the theoretical description of the AE signal formation processes in the framework of accepted concepts of materials destruction.

During the research, various models of the CM damage (destruction) processes are used. For example, discrete damage model [8-10], smeared damage model [11-13], and phase-field damage model [14-16].

One of the models for studying the destruction processes of composite materials is the model of representing the material in the form of a fiber bundle (FBM - fiber bundle model) [17-20]. The model considers the destruction of material which consists of a given number of fibers or cells. When an external load is applied to the material, its destruction is considered as a process of successive destruction of cells. It is assumed that each cell of the material is deformed elastically until it is destroyed, i.e. the loss of the bearing capacity of the composite material is due to the destruction of the cells that withstand the main load. The influence of matrix, the surface of interaction between matrix and binding substance, and also the interaction between cells on the processes of material destruction are not taken into account. The destruction process is analyzed according to the accepted rules of the redistribution of the applied load when the cell is destroyed on the remaining cells. Even load sharing (ELS), i.e. the applied load during cell destruction is evenly distributed between the remaining cells in the material. Local load sharing (LLS), i.e. the applied load at cell destruction is redistributed only to the nearest

(neighboring) cells.

In a majority of papers where the FBM model is used, the case of uniaxial tension is considered. In $[19,21]$, the case of transverse load application was considered. When considering the model, it was assumed that the CM cells can destruct upon reaching critical deformations (stresses) under bending, tension, or combined bending and tension. An analysis was carried out for some cases. In the first case, it was assumed that there are two independent methods of destruction, or the "OR rule". Moreover, taking into account the probabilities of threshold levels of destruction under tension and bending, an expression was obtained for changing the equivalent stress in the cells of material, as well as an expression for particular case of independent uniform distributions of threshold levels with boundaries [0, 1].

In the second case, von Mises fracture criterion was used. Several analytical and numerical patterns were obtained for changes in the number of remaining fibers with the development of the composite material destruction process. An expression was considered for the rate of $\mathrm{AE}$ energy release in $[18,22]$. However, there was not obtained an expression for the AE signal itself which was caused by a jump of the analyzed functions at the instant of complete destruction of the material.

Time dependencies for the cases of the "OR rule" and von Mises criterion were obtained for the number of remaining elements during destruction of the $\mathrm{CM}$ and generated $\mathrm{AE}$ signal [23-24]. In both cases, it was shown that with the development of destruction process, the time dependencies of changes in the remaining $\mathrm{CM}$ cells have a continuous character of decrease until complete destruction. In this case, continuous pulsed AE signals are formed. However, a comparison of the obtained patterns according to "OR rule" and von Mises criterion showed that the rate of increase in the equivalent stress on the $\mathrm{CM}$ elements by von Mises criterion is lower than according to the "OR" criterion [24]. In this case, the intensity of the CM cells destruction process by von Mises criterion is less than when using the "OR" criterion. This leads to increase of the destruction process duration by von Mises criterion, which leads to a decrease in the amplitude and duration of the generated AE signal. Time dependencies for the number of remaining elements during the $\mathrm{CM}$ destruction and for the generated AE signal were obtained in the form [24]:

$$
N(t)=N_{o} e^{-v_{0} \int_{t_{0}}^{t} e^{r\left[\sigma_{m}(t)-\sigma\left(t_{0}\right)\right] d t}},
$$

$$
U(t)=U_{0} v_{0}\left[\sigma_{m}(t)-\sigma\left(t_{0}\right)\right] \cdot e^{r\left[\sigma_{m}(t)-\sigma\left(t_{0}\right)\right]} \cdot e^{-v_{0} \int^{t} e^{r\left[\sigma_{m}(t)-\sigma\left(t_{0}\right)\right]} d t},
$$

where $\sigma_{m}(t), \sigma\left(t_{0}\right)$ are the change in the equivalent stress on the $\mathrm{CM}$ cells at linear input of deformation $\varepsilon=\alpha t$ ( $\alpha$ is the strain rate) and the threshold stress corresponding to the $\mathrm{CM}$ cells destruction onset time $t_{0}$, respectively; $U_{0}=N_{0} \beta \delta_{s}-$ maximum possible displacement at instant destruction of the
CM sample consisting of $N_{0}$ cells; $\beta-$ coefficient of proportionality between the fracture stress and the amplitude of a single disturbance pulse during destruction of a single cell; $\delta_{s}-$ parameter the numerical value of which is determined by the shape of a single disturbance impulse 
during destruction of a single cell; $v_{0}, r$ are constants depending on the physical and mechanical characteristics of the material.

The expressions for $\sigma_{m}(t), \sigma\left(t_{0}\right)$ are of the form

$$
\begin{gathered}
\sigma_{m}(t)=\alpha t \cdot 0.5\left[\left(2-2 \sqrt{\alpha t}+\alpha t^{\frac{3}{2}} \log ((1+\alpha t) /(1-\alpha t))\right)-\alpha t^{\frac{3}{2}}(2 \sqrt{(1-\sqrt{\alpha t}) / \alpha t}+\log ((1+\sqrt{1-\sqrt{\alpha t}}) /(1-\sqrt{1-\sqrt{\alpha t}})))\right] \\
\sigma\left(t_{0}\right)=\alpha t_{0} \cdot 0.5\left[\left(2-2 \sqrt{\alpha t_{0}}+\alpha t_{0}^{\frac{3}{2}} \log \left(\left(1+\alpha t_{0}\right) /\left(1-\alpha t_{0}\right)\right)\right)-\alpha t_{0}^{\frac{3}{2}}\left(2 \sqrt{\left(1-\sqrt{\alpha t_{0}}\right) / \alpha t_{0}}+\log \left(\left(1+\sqrt{1-\sqrt{\alpha t_{0}}}\right) /\left(1-\sqrt{1-\sqrt{\alpha t_{0}}}\right)\right)\right)\right]
\end{gathered}
$$

Expressions (1), (2), (3) and (4) include parameters that will affect the rate of the CM cells destruction process and the parameters of generated AE signals. One of these parameters is the rate of change in load application on the $\mathrm{CM}$ elements. Certainly, the subject of interest is the study of the effect of the loading rate on acoustic emission during the $\mathrm{CM}$ destruction with the use of von Mises criterion.

\section{Research Objectives and Results}

In this study of the $\mathrm{CM}$ destruction caused by transverse force with the use of von Mises criterion, the simulation outcomes of the CM cells destruction process and generated AE signals with increasing the strain rate will be considered. The time dependencies of the change in the number of remaining elements and the generated $\mathrm{AE}$ signals with increasing the strain rate will be obtained. The patterns of changes in the maximum amplitude of the generated $\mathrm{AE}$ signals and their duration with an increase in the CM strain rate will be determined and described.

As noted above, the expressions that describe the time dependencies of the change in the number of remaining cells during the $\mathrm{CM}$ destruction and the generated signal include parameters that will affect the rate of the CM cells destruction process and the parameters of the generated $\mathrm{AE}$ signals. One of these parameters is the CM strain rate.

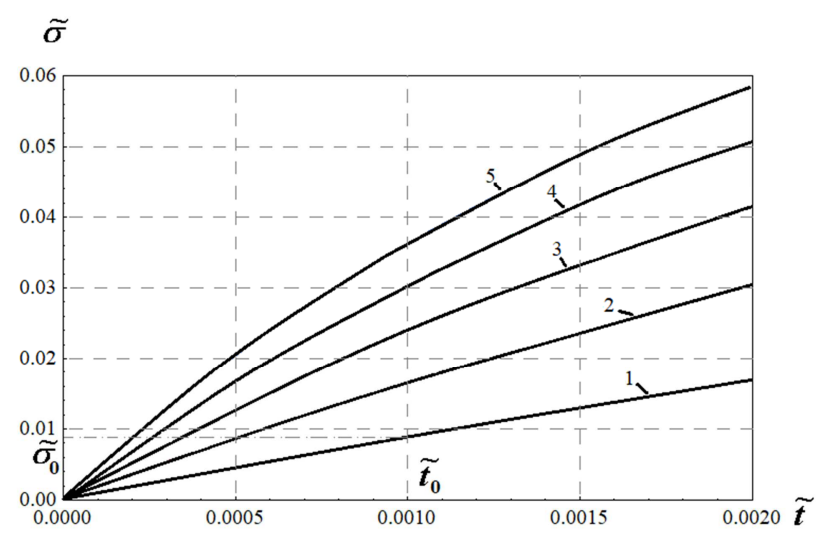

Figure 1. Time dependencies for the equivalent stress change, according to (3), at different rates $\tilde{\alpha}$ of the strain ramping: $1-\tilde{\alpha}=10 ; 2-\tilde{\alpha}=20 ; 3-$ $\tilde{\alpha}=30 ; 4-\tilde{\alpha}=40 ; 5-\tilde{\alpha}=50$.

Let us perform simulation the time dependencies of the change in the number of remaining cells during the $\mathrm{CM}$ destruction and the amplitude of the generated signal. The simulation will be carried out in relative units. When performing simulation, the values of the strain rate $\alpha$ will be changed in the range of values from $\tilde{\alpha}=10$ to $\tilde{\alpha}=50$ with increments of 10 . Figure 1 shows the calculation results, according to (3), for the dependency of the change in the equivalent stress at the $\mathrm{CM}$ cells upon linear input of deformation for the adopted simulation rates.

Figure 1 shows that with increasing the strain rate, the increase of stress variation rate on the CM cells is observed. Certainly, with an increase in the strain rate, the time to reach the threshold fracture stress of the CM cells will decrease.

When performing simulation the time dependencies of the number of remaining cells variation during the CM destruction, as well as the amplitude of the generated signal, we assume that at $\tilde{\alpha}=10$ the cells destruction onset time is $t_{0}$. This time corresponds to threshold stress $\sigma_{0}$. When performing simulation, the value of destruction onset time $t_{0}$ is taken to be $\widetilde{t_{o}}=0.001$. According to the calculations, see expression (3), for a given destruction onset time, the threshold stress $\sigma_{0}$ is $\widetilde{\sigma_{o}}=0.008897277688462064$.

We assume that at other strain rates the threshold stress $\widetilde{\sigma_{o}}$ does not change. Moreover, estimates suggest that with an increase in the strain rate, a decrease in the $\mathrm{CM}$ destruction onset time occurs: at $\tilde{\alpha}=20-\widetilde{t_{o}}=0.0005$; at $\tilde{\alpha}=30-\widetilde{t_{o}}=0.00033$; at $\tilde{\alpha}=40-\widetilde{t_{o}}=0.00025$; at $\tilde{\alpha}=50-\tilde{t_{o}}=0.0002$. The values $v_{0}$ and $\mathrm{r}$ in expressions (1) and (2), which characterize the stress-strain performance of the material, are assumed to be equal: $\widetilde{v_{o}}=100000 ; \tilde{r}=10000$. The choice of their magnitudes was considered in [24]. To compare the performed calculations of the destruction onset time with the results of calculations by the "OR" criterion considered in [23].

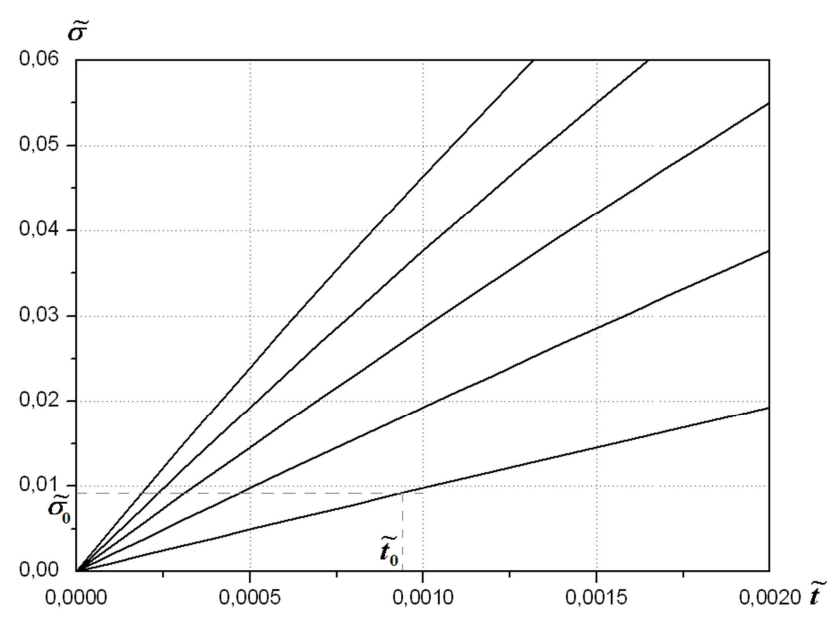

Figure 2. Time dependencies for the equivalent stress change, according to the "OR" criterion, for various rates of strain ramping: $1-\tilde{\alpha}=10 ; 2-\tilde{\alpha}=20$; $3-\tilde{\alpha}=30 ; 4-\tilde{\alpha}=40 ; 5-\tilde{\alpha}=50$.

The Figure 2 shows dependencies of the change in the 
equivalent stress on the CM cells according to the "OR" criterion for linear input of strain for the adopted simulation rates.

The performed estimates suggest that at $\widetilde{\sigma_{o}}=0.008897277688462064$ for the considered strain rates and according to the "OR" criterion (Figure 2), the CM cells destruction onset times will be: at $\tilde{\alpha}=10-\widetilde{t_{o}}=0.000906$; at $\tilde{\alpha}=20-\tilde{t_{o}}=0.00045$; at $\tilde{\alpha}=30-\tilde{t_{o}}=0.0003$; at $\tilde{\alpha}=40-$ $\widetilde{t_{o}}=0.00023$; at $\tilde{\alpha}=50-\widetilde{t_{o}}=0.00018$. It can be seen that, according to the "OR" criterion, a shift is observed toward smaller values of destruction onset time that is caused by a higher rate of reaching the threshold stress and, as a consequence, a higher rate of the $\mathrm{CM}$ cells destruction.

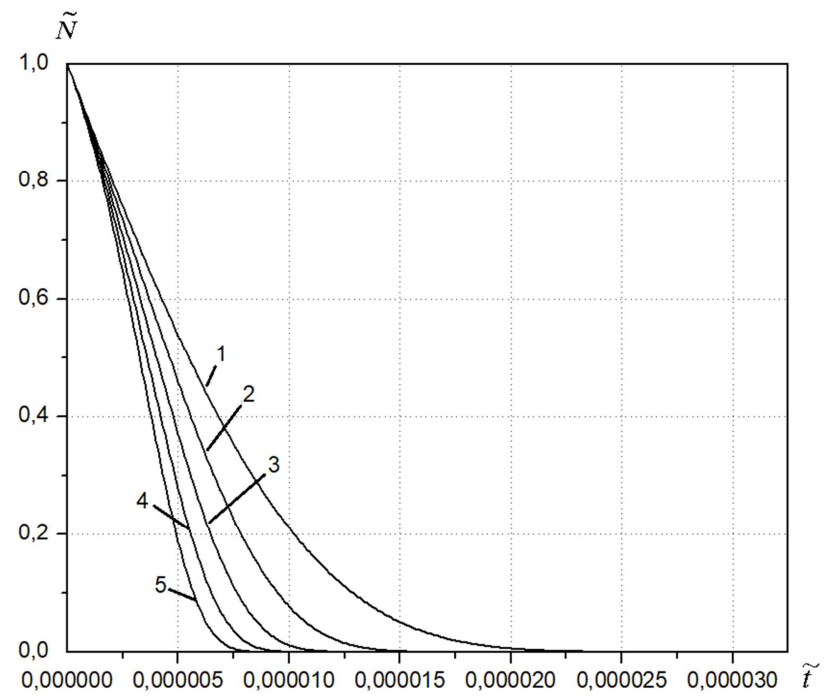

Figure 3. Plots of changes in the number of remaining elements, according to (1), in relative units during the composite material destruction under the action of transverse force. Strain rate: $1-\tilde{\alpha}=10 ; 2-\tilde{\alpha}=20 ; 3-\tilde{\alpha}=30 ; 4-$ $\tilde{\alpha}=40 ; 5-\tilde{\alpha}=50$. The destruction onset time: at $\tilde{\alpha}=20-\tilde{t_{0}}=0.0005$; at $\tilde{\alpha}=30$ $-\widetilde{t}_{o}=0.00033$; at $\tilde{\alpha}=40-\widetilde{t_{o}}=0.00025$; at $\tilde{\alpha}=50-\widetilde{t_{o}}=0.0002$.

Figure 3 shows the calculation data, according to (1), in the form of dependencies of the change in the number of remaining elements in relative units $\left(\widetilde{N}(t)=N(t) / N_{0}\right)$ for the accepted simulation conditions. The time in Figure 3 is reduced to the $\mathrm{CM}$ cells destruction onset time for each strain rate. The simulation outcomes (Figure 3) show that the curves of changes with time of the remaining CM cells have an accelerated fall pattern. The higher the strain rate, the higher the rate of decrease in time of the remaining cells number. In this case, the time of the CM cells destruction process decreases. Such a change in the rate of the CM cells destruction process should lead to a change in the parameters of the generated AE signals. Figure 4 shows the results of calculation, performed according to (2), for dependencies of the AE signal amplitude variation in relative units $\left(\widetilde{U}(t)=U(t) / U_{0}\right)$ at simulation conditions adopted above. When performing simulation, as before, the threshold stress of destruction onset at all strain rates was constant. In Figure 4 , time is reduced to the $\mathrm{CM}$ cells destruction onset time for each strain rate.

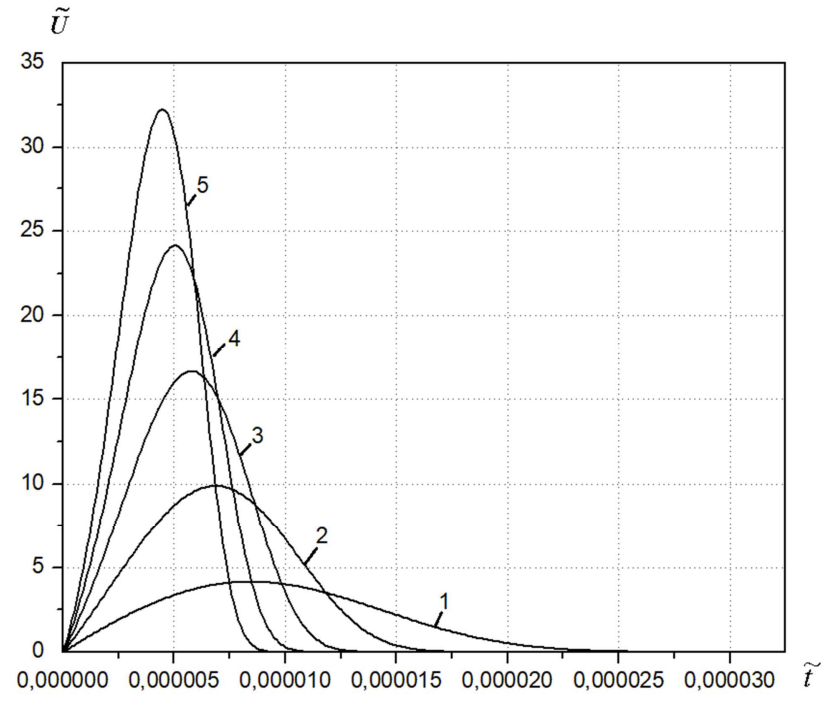

Figure 4. Plots of changes in the amplitude of AE signals, according to (2), in relative units during the CM cells destruction of under the action of a transverse force. Strain rate: $1-\tilde{\alpha}=10 ; 2-\tilde{\alpha}=20 ; 3-\tilde{\alpha}=30 ; 4-\tilde{\alpha}=40 ; 5-$ $\tilde{\alpha}=50 . \quad \widetilde{v_{o}}=100000, \quad r=10000 . \quad \widetilde{\sigma}_{o}=0.008897277688462064$. Destruction onset time: at $\tilde{\alpha}=20-\widetilde{t}_{o}=0.0005$; at $\tilde{\alpha}=30-\tilde{t}_{o}=0.00033$; at $\tilde{\alpha}=40-$ $\widetilde{t_{o}}=0.00025$; at $\tilde{\alpha}=50-\widetilde{t_{o}}=0.0002$.

The outcomes of AE signals simulation (figure 4) show that the generated AE signals are pulsed signals. An increase of the CM deformation rate leads to an increase of the amplitude of AE signal which is generated and a decrease of its duration. In this case, the transformations of the AE signal are observed. The shape of which approaches the triangular waveform gradually. Such a chance in the parameters of the generated AE signal is caused by rate increase and decaying of the lead time of the CM destruction process. An analysis of the results obtained in the form of dependencies of changes in the amplitude and duration of generated $\mathrm{AE}$ signals vs. increase of the CM strain rate is shown in figure 5.

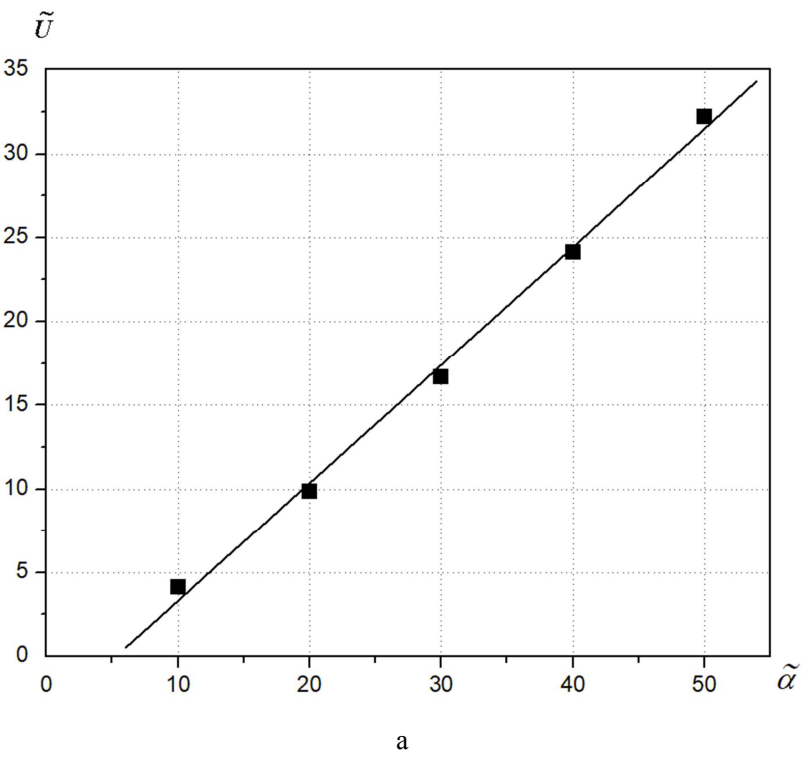




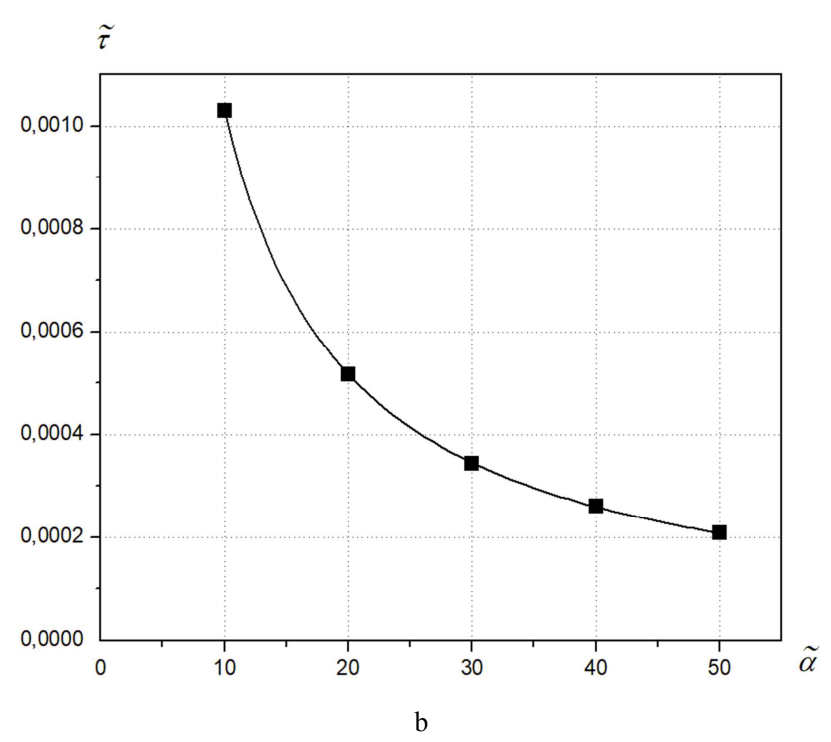

Figure 5. Dependencies of changes in the maximum amplitude (a) and duration (b) of $A E$ signals in relative units upon the CM cells destruction under the action of a transverse force vs. increase in the strain rate.

When plotting Figure 5 (b), in view of the asymptotic nature of the change in the trailing edge of the generated AE signals, their duration was determined from the fulfillment of the following condition for the amplitude ratio

$$
\widetilde{U}(\tilde{t}=\tilde{\tau})<<\widetilde{U}_{\max }\left(\tilde{t}=\tilde{\tau}_{0}\right),
$$

where $\tilde{\tau}$ is the duration of the AE signal; $\tilde{\tau}_{0}$ - the duration of the leading edge of the AE signal (corresponds to the maximum amplitude of the signal).

Moreover, for the duration of the AE signal, we can write

$$
\tilde{\tau}=\mathrm{m} \tilde{\tau_{o}},
$$

where $\mathrm{m}$ is an integer, which order of magnitudes is several units.

To estimate $\mathrm{m}$, we consider the ratio of signal amplitudes (5), according to the obtained data of simulating AE signals for various values of $\mathrm{m}$. So, at $\tilde{\alpha}=10$ and $\mathrm{m}=3$, the amplitude ratio is 0.00643 , and at $\mathrm{m}=4$ this ratio is already equal to $1.24 \cdot 10^{-5}$. At $\tilde{\alpha}=20$ and $\mathrm{m}=2.5$, the amplitude ratio is 0.001982 , and at $\mathrm{m}=2.6$ this ratio is already equal to $9.24 \cdot 10^{-7}$ At $\tilde{\alpha}=30$ and $\mathrm{m}=2.3$, the amplitude ratio is 0.001471 , and at $\mathrm{m}=2.5$ this ratio is already equal to $3.82 \cdot 10^{-5}$. At $\tilde{\alpha}=40$ and $\mathrm{m}=2.1$, the amplitude ratio is 0.00213 , and at $\mathrm{m}=2.2$ this ratio is already equal to $6.47 \cdot 10^{-5}$. At $\tilde{\alpha}=50$ and $\mathrm{m}=2$, the amplitude ratio is 0.001851 , and at $\mathrm{m}=2.2$ this ratio is already equal to $2.72 \cdot 10^{-5}$. Based on the estimates obtained, curve 1 in Figure 4 (b) was constructed under the condition that the smallness of the amplitude ratios is limited by the third order of magnitude.

The results show that, with a linear strain rate increase, the dependency of the AE signal maximum amplitude change on the strain rate has a linear character of increase (Figure 5 (a)). The dependency is well presented by function

$$
\widetilde{U}=-3.7184+0.7043 \tilde{\alpha}
$$

When describing the dependency shown in Figure 4 (a), by expression (7), the value of correlation coefficient $\mathrm{R}$ was: $\mathrm{R}=0.99785$. The residual standard deviation was 0.84426 .

With a linear increase of the strain rate, the dependency of the AE signal duration on the strain rate has a non-linear nature of decrease (Figure 5 (b)). Dependency is well presented by function

$$
\tilde{\tau}=0.01013 \tilde{\alpha}^{-0.99292} \text {. }
$$

When describing the dependency shown in Figure 5 (b) by expression (8), the coefficient of determination of $\mathrm{R}^{2}$ was: $\mathrm{R}^{2}=0.99999$. In this case, the residual dispersion was $2.077 \cdot 10^{-12}$.

The research results show that the increase of the $\mathrm{CM}$ strain rate leads to increase of the rate of change of equivalent stresses, which are calculated by von Mises criterion. At a given constant threshold stress of the CM cells destruction onset, an increase in the strain rate leads to an increase of the rate and a decrease of the lead time of the CM cells destruction. The AE signals formed during the CM cells destruction are the pulsed signals.

In this case, an increase of the $\mathrm{CM}$ deformation rate leads to an increase of the amplitude of the generated AE signal and a decrease of its duration. The decrease of the signal duration is caused by decrease of the lead time of the CM cells destruction.

A gradual transformation of the AE signal into a triangular signal is also observed. The dependency of the change in the maximum amplitude of the AE signal from the strain rate is a linear increase. At the same time, the dependency of the change of the $\mathrm{AE}$ signal duration from the strain rate is a nonlinear decrease.

\section{Summary}

The simulation outcomes of the CM cells destruction process by von Mises criterion, as well as generated $\mathrm{AE}$ signals at increase in the strain rate are considered. The time dependencies of the change in the number of remaining elements are obtained during development of the destruction process and generated $\mathrm{AE}$ signals for various $\mathrm{CM}$ deformation rates. It is shown that at increase in the $\mathrm{CM}$ deformation rate, there occurs an increase in the rate of fall of the curves that characterize change in time of remaining $\mathrm{CM}$ cells. In this case, a decrease in time of the process of the CM cells destruction is observed. Such an increase in the rate of fall of the curves describing the changes in time of the remaining CM cells and decreasing duration of the $\mathrm{CM}$ cells destruction process is caused by increase in the rate of the $\mathrm{CM}$ cells destruction process.

It was determined that at increasing the strain rate, the amplitude of the generated AE signal increases. In this case, a decrease in their duration is observed. It is also shown that with an increase in the $\mathrm{CM}$ deformation rate, the AE signal gradually transforms into a triangular waveform. Such a change in the parameters of the generated AE signals is due to an increase in the rate of the CM cells destruction. Regularities of change in the maximum amplitude of the generated AE signals and their duration at increasing the CM strain rate are obtained and described. It is shown that at 
increasing the $\mathrm{CM}$ strain rate, the dependency of change in the AE signals maximum amplitude is well described by a ramp function. In this case, the dependency of change in the AE signals duration on increase in the strain rate has a nonlinear nature of decrease and it is well described by an exponential function. In further works, it is of interest to study the effect of the CM stress-strain performance on destruction processes and parameters of generated AE signals

\section{References}

[1] Bussiba A. Damage evolution and fracture events sequence in various composites by acoustic emission technique /A. Bussiba, M. Kupiec, S. Ifergane, R. Piat, T. Böhlke // Composites Science and Technology. -2008.-vol. 68.-P. 1144-1155.

[2] Nimdum P. Use of acoustic emission to discriminate damage modes in carbon fibre reinforced epoxy laminate during tensile and buckling loading / P. Nimdum, J. Renard // ECCM 15 - 15th European Conference on Composite Materials, Jun 2012, Venise, Italy.- 2012.- 8 p., hal-01109533.

[3] Cluster Analysis of Acoustic Emission Signals for Carbon/Epoxy Composite in Four-Point Bending Test / R. Khamedi, O. Pedram // International Journal of Engineering: TRANSACTIONS C: Aspects.-2015.-vol. 28,-No. 9.-P. 1336-1342.

[4] Ereifej N. S. Fracture of fiber-reinforced composites analyzed via acoustic emission / N. S. Ereifej, Y. G. Oweis, S. K. Altarawneh // Dental Materials Journal.-2015.-vol. 34. No. 4.-P. 417-424.

[5] Fotouhi M. Detection of fibre fracture and ply fragmentation in thin-ply UD carbon/glass hybrid laminates using acoustic emission / M. Fotouhi, P. Suwarta, M. Jalalvand, G. Czel, M. R. Wisnom // Composites: Part A. 2016.-vol. 86,-P. 66-76.

[6] Gholizadeh S. Glass fiber-reinforced polyester composite fatigue crack monitoring using acoustic emission / S. Gholizadeh, Z. Leman, B. Baharudin, O. Innayatullah // International Journal of Advances in Science Engineering and Technology, 2016.-vol. 4. No 2.-P. 100-105.

[7] Godin N. Damage diagnostic and lifetime prognosis for ceramic matrix composite with acoustic emission during longterm mechanical tests at intermediate temperature / N. Godin, P. Reynaud, G. Fantozzi // EWGAE. 33-rd European Conference on Acoustic emission testing (12-14 September, 2018, Senlis, France). -2018.-16 p.

[8] Moure M. M., Sánchez-Sáez S., Barbero E. \& Barbero E. J. (2014). Analysis of damage localization in composite laminates using a discrete damage model / M. M. Moure, S. Sánchez-Sáez, E. Barbero, E. J. Barbero // Composites: Part B.-2014.-vol. 66.-P. 224-232.

[9] Barbero E. J. Determination of material parameters for discrete damage mechanics analysis of carbon-epoxy laminates / E. J. Barbero, F. A. Cosso // Composites Part B.2014.-vol. 56.-P. 638-646.

[10] Jin W. Micromechanics based discrete damage model with multiple non-smooth yield surfaces: Theoretical formulation, numerical implementation and engineering applications / W.
Jin, C. Arson // International Journal of Damage Mechanics, 27 (5): 611-639, 2018.

[11] Oliver J. A consistent characteristic length for smeared cracking models / J. Oliver // International Journal for Numerical Methods in Engineering.-1989.-vol. 28.-No 2.-P. 461-474.

[12] Wisnom M. R., Modelling discrete failures in composites with interface elements / M. R. Wisnom // Composites Part A Applied Science and Manufacturing.-2010.-vol. 41.-No. 7.-P. 795-805.

[13] Heinrich C. Investigation of progressive damage and fracture in laminated composites using the smeared crack approach / C. Heinrich, A. M. Waasy // 53rd AIAA/ASME/ASCE/AHS/ASC Structures, Structural Dynamics and Materials Conference (23 - 26 April 2012, Honolulu, Hawaii).-2012.-19 p.

[14] McAuliffe C. A coupled phase field shear band model for ductile brittle transition in notched plate impacts / C. McAuliffe, H. Waisman // Computer Methods in Applied Mechanics and Engineering.-2016.-vol. 305.-P. 173-195.

[15] Bleyer J. Phase-field modelling of anisotropic brittle fracture including several damage mechanisms / J. Bleyer, R. Alessi // Computer Methods in Applied Mechanics and Engineering.vol. 336.-P. 213-236.

[16] Dzepina B. A phase field model of pressure-assisted sintering / B. Dzepina, D. Balinta, D. Dini // Journal of the European Ceramic Society.-2019.-vol. 39.-P. 173-182.

[17] Newman W. I. Time dependent fiber-bundles with local load sharing / W. I. Newman, S. L. Phoenix // Phys. Rev. E.-2001.v. $63 .-021507$.

[18] Turcotte D. L. Micro and macroscopic models of rock fracture / D. L. Turcotte, W. I. Newman, R. Shcherbakov // Geophys. J. Int.-2003.-vol. 152.-P. 718-728.

[19] Kun F. Extension of fibre bundle models for creep rupture and interface failure / F. Kun, R. C. Hidalgo, F. Raischel, H. J. Herrmann // International Journal of Fracture.-2006.-vol. 140.No 1-4.-P. 255-265.

[20] Swolfs Y. Global load-sharing model for unidirectional hybrid fibre-reinforced composites / Y. Swolfs, R. M. McMeeking, V. P. Rajan, F. W. Zok, I. Verpoest, L. Gorbatikh // Journal of the Mechanics and Physics of Solids. -2015.-vol. 84.-P. 380-394.

[21] Raischel F. A simple beam model for the shear failure of interfaces / F. Raischel, F. Kun, H. J. Herrmann // Phys. Rev. E.-2005.-vol. 72.-P. 046126.

[22] Shcherbakov R. On modeling of geophysical problems: A dissertation for degree of doctor of philosophy / Robert Shcherbakov. - Cornell university, -2002.-209 P.

[23] Filonenko S. Destruction of composite material by shear load and formation of acoustic emission / S. Filonenko, V. Kalita, A. Kosmach // Aviation.-2012.-v. 16.-No 1.-P. 5-13.

[24] Filonenko S. A study to determine the onset of catastrophic wear of a processing tool by statistical parameters of acoustic emission / Stakhova A. // Eastern-European Journal of Enterprise Technologies. 2019.-v. 6, No 9 (102). 